\title{
A case report of exogenous lipoid pneumonia associated with avocado/ soybean unsaponifiables
}

\author{
Jacques BOUTROS ${ }^{1}$, Marine MUZZONE${ }^{1}$, Jonathan BENZAQUEN ${ }^{1,2}$, Michael LEVRAUT ${ }^{1}$, \\ Charles-Hugo MARQUETTE ${ }^{1,2^{*}}$ (D), Fanny ROCHER ${ }^{3}$, Yann DIASCORN ${ }^{4}$, Bernard PADOVANI ${ }^{4}$, \\ Véronique HOFMAN ${ }^{5}$ and Sylvie LEROY ${ }^{1,6}$
}

\begin{abstract}
Background: Exogenous lipoid pneumonia is a rare disease resulting from intra-alveolar accumulation of lipids of mineral, vegetal, or animal origin, that induce a foreign body type of inflammatory reaction in the lungs. Gastroesophageal reflux disease and other esophageal abnormalities have often been associated with this disease.

Case presentation: We herein report the case of an 83-year-old patient in whom a follow-up chest computed tomography scan, for a lingular consolidation, showed multifocal ground glass and consolidative opacities with areas of low attenuation, suggestive of exogenous lipid pneumonia. The patient had been on piascledine capsules (avocado/soybean unsaponifiables) for 20 years and had a hiatal hernia with documented gastroesophageal reflux disease. After thorough history taking, no other predisposing factors were found. The diagnosis was confirmed using oil red staining of bronchoalveolar lavage showing lipid-laden macrophages and extracellular lipid droplets.

Conclusions: To our knowledge, this is the first case of ELP secondary to avocado/soybean unsaponifiables in the literature.
\end{abstract}

Keywords: Exogenous lipoid pneumonia, Avocado oil, Soybean oil, Hiatal hernia, Gastroesophageal reflux disease

\section{Background}

Exogenous lipoid pneumonia (ELP) is a rare lung condition due to the inflammatory reaction generated by the presence of foreign fatty substances in the alveoli. ELP can be suspected when a clinical history of inhalation or aspiration of fatty substances and chest imaging are compatible [1-3]. Confirmation of the diagnosis requires special staining of bronchoalveolar lavage (BAL) fluid or lung biopsies $[4,5]$. We present an unusual case of ELP caused by avocado/ soy unsaponifiables (ASU).

\footnotetext{
* Correspondence: marquette.c@chu-nice.fr

1 Department of Pulmonary Medicine, Université Côte d'Azur, CHU de Nice, FHU OncoAge, 30 avenue de la voie Romaine, CS51069, 06001 Nice, France 2Université Côte d'Azur, CNRS UMR7284, Inserm U1081, Institute of Research on Cancer and Ageing (IRCAN), Nice, France

Full list of author information is available at the end of the article
}

\section{Case presentation}

An 83-year old never-smoking female consulted in 2017 for a dry nocturnal cough, without dyspnea. An incidental lingular consolidation had been detected on a chest computed tomography (CT) scan in 2010, and was followed by regular chest CTs and positron emission tomography- computed tomography (PET/CT) scans until 2016. She had had two undiagnostic bronchoscopies and refused further invasive examinations including thoracoscopy. She had a medical history of osteoarthritis and hiatal hernia. Until her visit in 2017, she was in good general condition, had no signs of respiratory disease, and her physical examination was normal. The PET/CT scan (Fig. 1) performed in 2015 showed no abnormal metabolic activity. The follow-up PET-CT (Fig. 1) performed 15 months later showed a slight increase in size of the consolidation $(20 \mathrm{~mm})$ without abnormal uptake.

(c) The Author(s). 2019 Open Access This article is distributed under the terms of the Creative Commons Attribution 4.0 International License (http://creativecommons.org/licenses/by/4.0/), which permits unrestricted use, distribution, and 


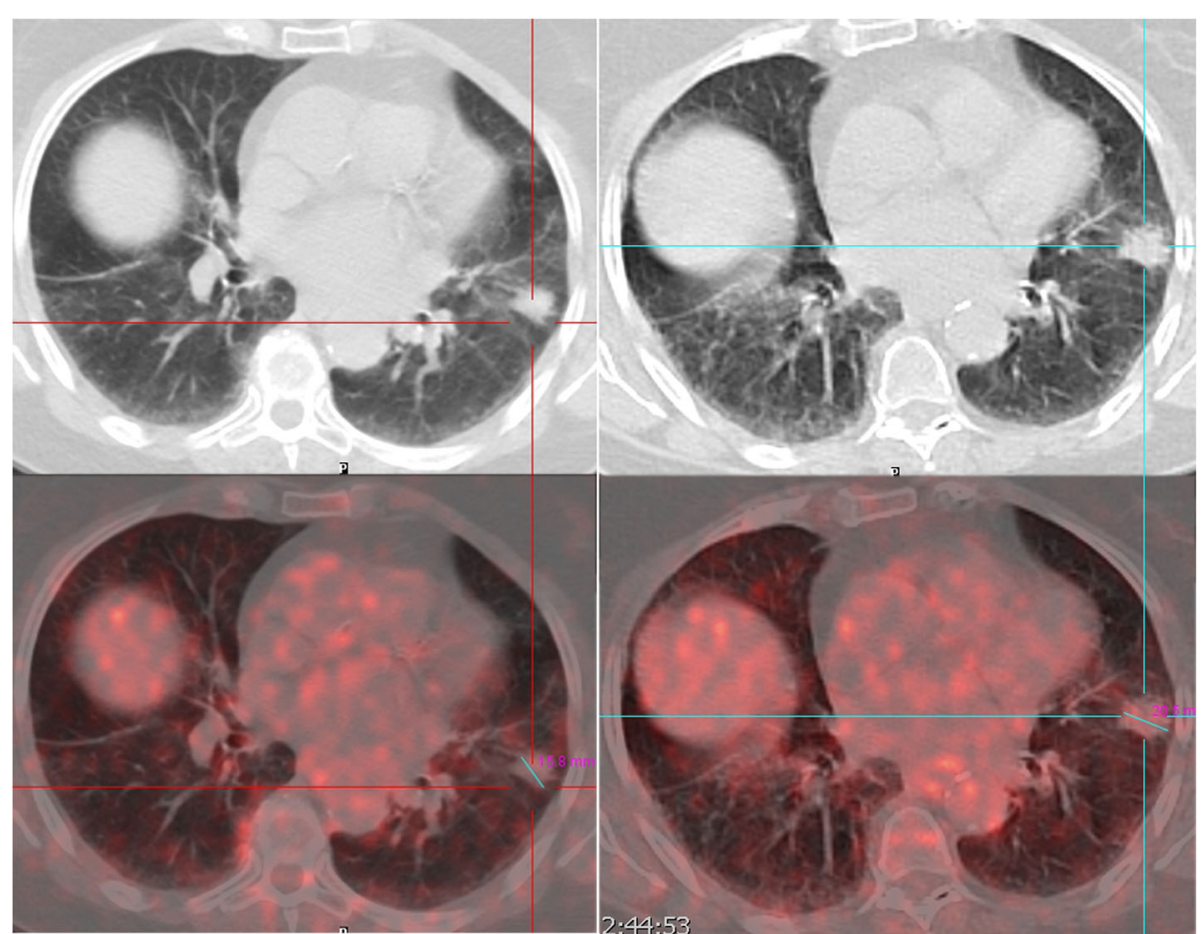

Fig. 1 Left: baseline PET scanner, $15.6 \mathrm{~mm}$ lingular consolidation, no abnormal FDG uptake; right: follow up PET scanner, 15 months later, 20.5 $\mathrm{mm}$ lingular consolidation, no abnormal FDG uptake

When she presented in 2017, the chest examination was still normal. Pulmonary function tests (PFTs) showed the onset of a restrictive disease with 3.69 Liters (75\% of predicted) of total lung capacity versus $5.19 \mathrm{Li}-$ ters 1 year earlier, without weight gain. No oxygen desaturation was detected at rest or during a 6-min walking test. This time, a CT scan showed multifocal groundglass and consolidative opacities with heterogeneous densities, including areas of low attenuation, indicative of intrapulmonary lipid, in the left upper lobe (LUL) (Fig. 2). After thorough history taking concerning exposure to fatty substances, the patient denied any form of

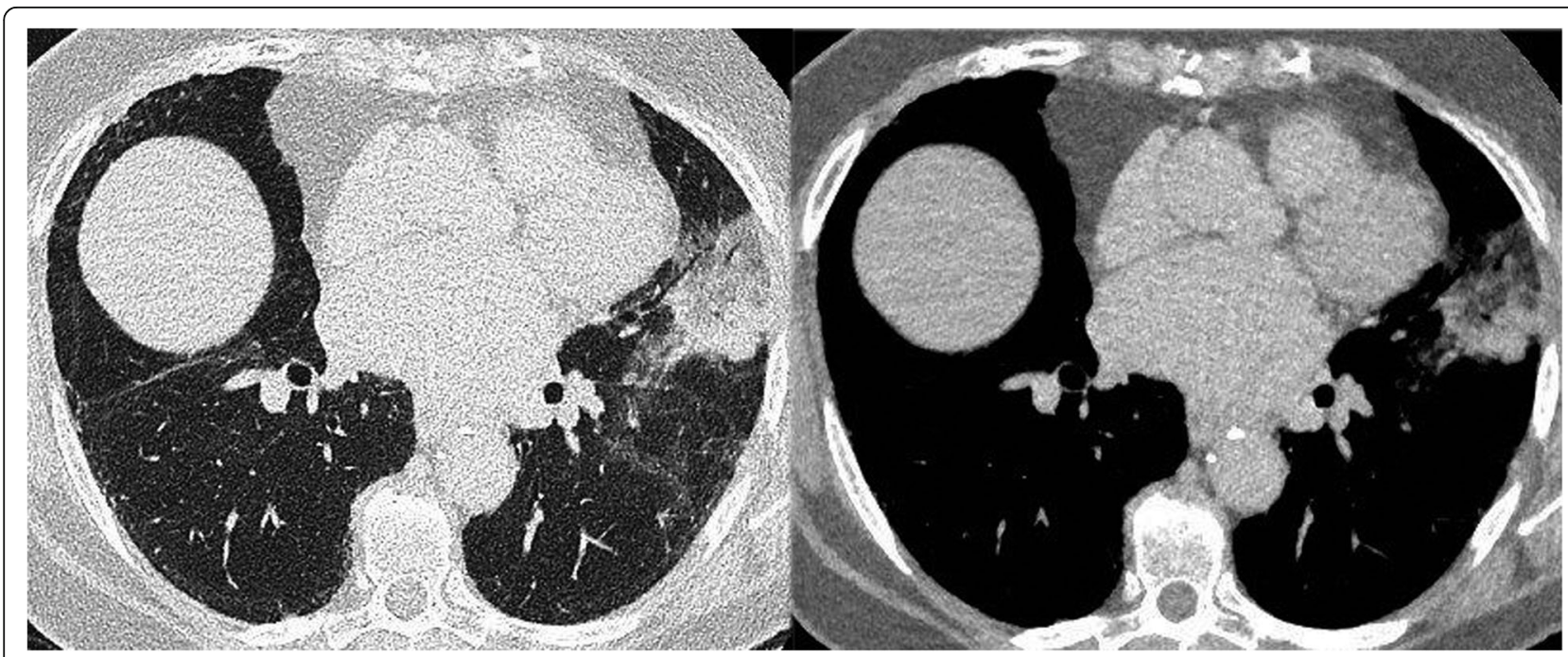

Fig. 2 Follow-up TCT scan in 2018 showing lingular consolidation with multifocal ground-glass opacities in the lingular and culminal segments (left) and areas of fat attenuation (right) 
inhalation of volatile hydrocarbons. However, she had been on ASU twice daily for 20 years to treat osteoarthritis. Gastroesophageal reflux disease (GERD) was documented using 24-h pH-metry showing 90 acidreflux episodes per $24 \mathrm{~h}$, despite treatment with esomeprazole. Bronchoscopy was performed, and the BAL fluid was macroscopically turbid white. Oil red $\mathrm{O}$ staining of BAL showed lipid-laden macrophages (Fig. 3) and extracellular lipid droplets. Cultures of the BAL were negative.

After confirmation of the diagnosis, in the absence of other fatty-substance exposure, a presumptive diagnosis of ELP caused by ASU aspiration in a patient with GERD was established. This treatment was stopped. No surgical treatment of her hiatal hernia was suggested in this patient who had refused invasive procedures. One year later, the patient was clinically stable, her cough regressed and her PFTs normalized. Her chest CT showed persistent lingular consolidation and regression of the ground-glass opacities.

\section{Discussion and conclusions}

ELP is an unusual form of pneumonitis, caused by exogenous lipids of mineral, vegetal, or animal origin [6], reaching the alveoli by inhalation of volatile hydrocarbons [7-9], or by aspiration of oil-based substances. Most cases of ELP result from mineral oil aspiration found in laxatives and oily nose drops [1].

The risk of ELP is increased in multiple esophageal disorders and GERD $[1,4,10]$. Hiatal hernia has been associated with ELP in 2 cases reported in the literature $[11,12]$.

Diagnosing ELP is based on a history of exposure to exogenous fat, compatible radiological findings, and a demonstration of lipids in lung biopsy specimens or BAL $[1,6,10,13,14]$. In a French case series, GERD was associated with ELP in $50 \%$ of cases [1]. Lipids tend to overlay gastrointestinal fluids in static situations, making patients more vulnerable to chronic aspiration of fat, especially in hiatal hernia with GERD [12]. Our patient had typical ELP with a compatible CT scan and BAL. Although it has never been described in the literature, the most probable culprit in this context was the $300 \mathrm{mg}$ non-gastro-resistant capsules of ASU capsules [15-17]. We consulted the international databases of "Expanscience Laboratoires", the manufacturer of the drug, looking for similar adverse effect in the drug safety databases, but we did not find any. Although the patient's cough may arguably have been due to the ELP itself, its nocturnal timing and the GERD-compatible 24-h pH-metry suggested symptomatic nocturnal GERD.

ELP is often insidious, and in our patient, the initial lingular opacity could be an inaugural sign of the disease [2], even though no low-attenuation areas were seen within it. Both PET scans did not show any abnormal uptake. We currently do not know the value of PET scans in ELP evaluation. Nonetheless, several papers have reported cases of ELP mimicking neoplastic nodules on PET-CT, with moderate uptake of up to 4.4 of SUVmax [18-20].

Although ELP is uncommon, the pneumologist should have a high degree of suspicion whenever a history and imaging results are suggestive of the disease. Thorough search of the culprit source is essential to reverse the disease. Our case is the first to report ELP caused by ASU, readily commercialized as dietary joint supplements.

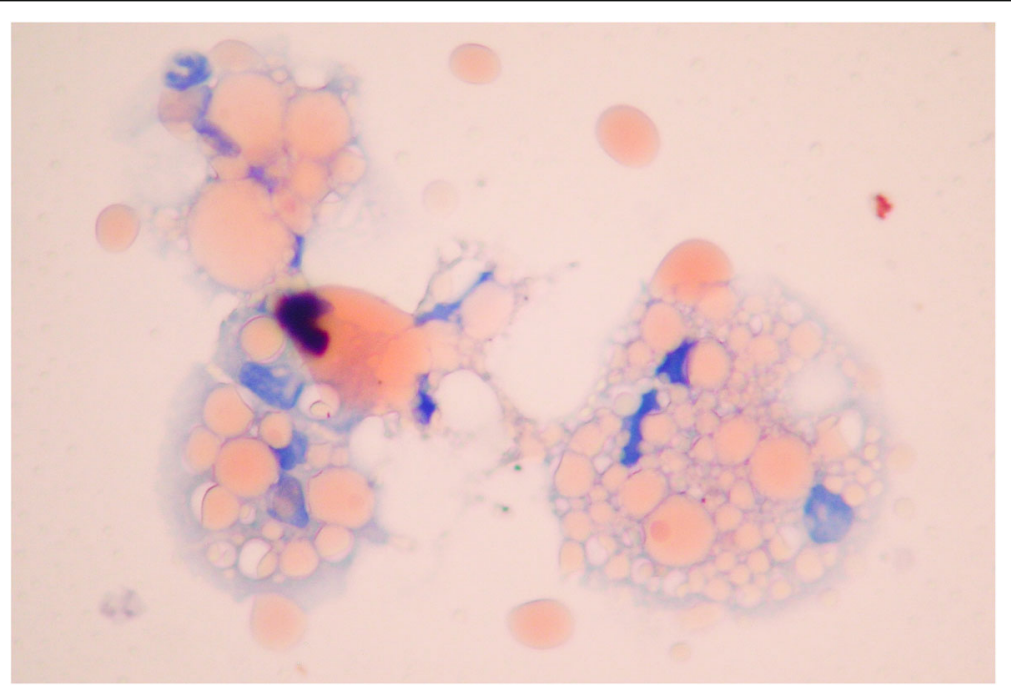

Fig. 3 Alveolar macrophages in bronchoalveolar lavage with intracytoplasmic micro and macrovacuoles filled with lipid associated with extracellular lipid droplets (oil red, original magnification $\times 1000$ ) 


\section{Case Report Timeline}

\begin{tabular}{|c|c|c|c|c|}
\hline 2010 & April 2015 & June 2016 & $\begin{array}{l}\text { December } \\
\text { 2017-March } \\
2018\end{array}$ & March 2019 \\
\hline $\begin{array}{l}\text { Chest CT: } \\
\text { lingular } \\
\text { consolidation }\end{array}$ & $\begin{array}{l}\text { Follow-up } \\
\text { PET/CT scan: } \\
\text { non- } \\
\text { hypermeta- } \\
\text { bolic lingular } \\
\text { consolidation } \\
\text { Pulmonary } \\
\text { function } \\
\text { tests: normal }\end{array}$ & $\begin{array}{l}\text { Follow-up } \\
\text { PET/CT scan: } \\
\text { persistant } \\
\text { non- } \\
\text { hypermeta- } \\
\text { bolic lingular } \\
\text { consolidation }\end{array}$ & $\begin{array}{l}\text { Clinically: } \\
\text { dyspnea and } \\
\text { cough } \\
\text { Chest CT: } \\
\text { lingular } \\
\text { consolidation } \\
\text { compatible } \\
\text { with lipoid } \\
\text { pneumonia } \\
\text { Bronchoscopy } \\
\text { with broncho- } \\
\text { alveolar lav- } \\
\text { age: } \\
\text { compatible } \\
\text { with lipoid } \\
\text { pneumonia } \\
\text { Pulmonary } \\
\text { function test: } \\
\text { restrictive } \\
\text { pattern. } \\
\text { pH-metry: } \\
\text { compatible } \\
\text { with GERD } \\
\text { Decision: stop } \\
\text { treatment with } \\
\text { avocado/ } \\
\text { soybean } \\
\text { unsaponifiables }\end{array}$ & $\begin{array}{l}\text { Clinically: } \\
\text { improvement } \\
\text { Chest CT: } \\
\text { regression of } \\
\text { ground-glass } \\
\text { opacities. Per- } \\
\text { sistence of lin- } \\
\text { gular consoli- } \\
\text { dation Pul- } \\
\text { monary func- } \\
\text { tion test: } \\
\text { normal }\end{array}$ \\
\hline
\end{tabular}

\section{Abbreviations}

ASU: Avocado/soy unsaponifiable; BAL: Bronchoalveolar lavage; CT: Computed tomography; ELP: Exogenous lipoid pneumonia; GERD: Gastroesophageal reflux disease; LUL: Left upper lobe; PET/CT: Positron emission tomography - computed tomography; PFTs: Pulmonary function tests

\section{Acknowledgements}

Not applicable.

\section{Authors' contributions}

$J B 1, M M, J B 2, M L C H M, Y D, B P, V H$ and SL were involved in the clinical care of the patient. FR was responsible for the pharmacovigilance investigation. All contributed to and approved of the final manuscript. The datasets used and/or analyzed during the current study available from the corresponding author on reasonable request.

\section{Funding}

None.

\section{Availability of data and materials}

The datasets used and/or analyzed during the current study are available from the corresponding author on reasonable request.

\section{Ethics approval and consent to participate}

N/A

\section{Consent for publication}

Written informed consent for publication of their personal or clinical details and/or clinical images was obtained from the patient.

\section{Competing interests}

The authors declare that they have no competing interests.

\section{Author details}

'Department of Pulmonary Medicine, Université Côte d'Azur, CHU de Nice, FHU OncoAge, 30 avenue de la voie Romaine, CS51069, 06001 Nice, France. 2Université Côte d'Azur, CNRS UMR7284, Inserm U1081, Institute of Research on Cancer and Ageing (IRCAN), Nice, France. ${ }^{3}$ Université Côte d'Azur, CHU de
Nice, Regional Pharmacovigilance Center, Nice, France. ${ }^{4}$ Department of Radiology, Université Côte d'Azur, CHU de Nice, Nice, France. ${ }^{5}$ Laboratory of Clinical and Experimental Pathology, Université Côte d'Azur, CHU de Nice, FHU OncoAge, Nice, France. ${ }^{6}$ Université Côte d'Azur, CNRS UMR 7275 Institut de Pharmacologie Moléculaire et Cellulaire, Sophia Antipolis, France.

Received: 26 March 2019 Accepted: 14 November 2019

Published online: 03 December 2019

\section{References}

1. Gondouin A, Manzoni P, Ranfaing E, Brun J, Cadranel J, Sadoun D, et al. Exogenous lipid pneumonia: a retrospective multicentre study of 44 cases in France. Eur Respir J. 1996;9(7):1463-9.

2. Betancourt SL, Martinez-Jimenez S, Rossi SE, Truong MT, Carrillo J, Erasmus JJ. Lipoid pneumonia: Spectrum of clinical and radiologic manifestations. Am J Roentgenol. 2010;194(1):103-9.

3. Laurent F, Philippe JC, Vergier B, Granger-Veron B, Darpeix B, Vergeret J, et al. Exogenous lipoid pneumonia: HRCT, MR, and pathologic findings. Eur Radiol. 1999;9(6):1190-6.

4. Hadda V, Khilnani GC. Lipoid pneumonia: an overview. Expert Rev Respir Med. 2010;4(6):799-807.

5. Spickard A. Exogenous Lipoid Pneumonia. Arch Intern Med. 1994;154(6):686.

6. Marchiori E, Zanetti G, Mano CM, Hochhegger B. Exogenous lipoid pneumonia. Clinical and radiological manifestations. Respir Med. 2011;105(5):659-66.

7. Kilaru H, Prasad S, Radha S, Nallagonda R, Kilaru SC, Nandury EC. Nasal application of petrolatum ointment - a silent cause of exogenous lipoid pneumonia: successfully treated with prednisolone. Respir Med Case Rep. 2017;22:98-100.

8. Cherrez Ojeda I, Calderon JC, Guevara J, Cabrera D, Calero E, Cherrez A. Exogenous lipid pneumonia related to long-term use of Vicks VapoRub by an adult patient: a case report. BMC Ear, Nose and Throat Disorders . 2016 Jan [cited 2018 Dec 19];16(1). Available from: http://bmcearnosethroatdisord. biomedcentral.com/articles/10.1186/s12901-016-0032-6

9. Madan K, Walia R, Kumar J, Jain D, Guleria R, Venkatnarayan K. "Diesel siphoner's lung": Exogenous lipoid pneumonia following hydrocarbon aspiration. Lung India. 2014:31(1):63.

10. Rabahi MF, Ferreira AA, Madeira JGP, Galvao PM, Pinto SA. Pneumonia lipoide secundária ao uso prolongado de óleo de prímula. J Bras Pneumol. 2010;36(5):657-61.

11. Steinberg I. Lipoid pneumonia associated with Paraesophageal hernia: Angiocardiographic study of a case. Diseases of the Chest. 1960;37(2):157-62

12. Nguyen CD, Oh SS. A Case of Exogenous Lipoid Pneumonia. Respir Care. 2013;58:23-7.

13. Knauer-Fischer $\mathrm{S}$, Ratjen F. Lipid-laden macrophages in bronchoalveolar lavage fluid as a marker for pulmonary aspiration. Pediatr Pulmonol. 1999;27(6):419-22.

14. Marchiori E, Zanetti G, Nobre LF, Takayassu TC, Irion KL. Lipoid pneumonia complicating Megaesophagus secondary to Chagas disease: high-resolution computed tomography findings. J Thorac Imaging. 2010;25(2):179-82.

15. Olivier $P$, Montastruc J-L. Profil d'effets indésirables des insaponifiables d'avocat et de soja. Presse Med. 2010;39(10):e211-6.

16. Pavelka K, Coste P, Géher P, Krejci G. Efficacy and safety of piascledine 300 versus chondroitin sulfate in a 6 months treatment plus 2 months observation in patients with osteoarthritis of the knee. Clin Rheumatol. 2010 Jun;29(6):659-70.

17. Cameron M, Chrubasik S. Oral herbal therapies for treating osteoarthritis. Cochrane Musculoskeletal Group, editor. 2014Cochrane Database of Systematic Reviews [Internet]. 22 [cited 2019 Aug 18]; Available from: http:// doi.wiley.com/10.1002/14651858.CD002947.pub2

18. Chardin D, Nivaggioni G, Viau P, Butori C, Padovani B, Grangeaon C, et al. False positive 18FDG PET-CT results due to exogenous lipoid pneumonia secondary to oily drug inhalation. Medicine (Baltimore) . 20172 [cited 2018 Dec 19];96(22). Available from: https://www.ncbi.nlm.nih.gov/pmc/articles/PMC5459704/

19. Talwar A, Mayerhoff R, London D, Shah R, Stanek A, Epstein M. False-Positive PET Scan in a Patient With Lipoid Pneumonia Simulating Lung Cancer. Clin Nucl Med. 2004;29(7):426-8.

20. Mokhlesi B, Angulo-Zereceda D, Yaghmai V. False-positive FDG-PET scan secondary to lipoid pneumonia mimicking a solid pulmonary nodule. Ann Nucl Med. 2007;21(7):411-4.

\section{Publisher's Note}

Springer Nature remains neutral with regard to jurisdictional claims in published maps and institutional affiliations. 